\title{
A weak scattering model for tone haystacking
}

\author{
A. McAlpine, C.J. Powles and B.J. Tester \\ Institute of Sound and Vibration Research, University of Southampton, Southampton, SO17 1BJ, UK
}

\begin{abstract}
The scattering of sound by turbulence in a jet shear layer is considered. Spectral broadening or 'haystacking' is the process whereby the turbulent, timevarying inhomogeneities in the flow scatter tonal sound fields, which decreases the level of the incident tone, but increases the broadband level around the frequency of the tone. The scattering process is modelled analytically, using high-frequency asymptotic methods and a weak-scattering assumption. Analytical models for the far-field spectral density of the scattered field are derived for two cases: (1) any polar angle including inside the cone of silence; (2) polar angles outside the cone of silence. At polar angles outside the cone of silence, the predictions from the two models are very similar, but using the second model it is considerably simpler to evaluate the far-field spectral density. Simulation results are compared to experimental data, albeit only at a polar angle of $90^{\circ}$. The model correctly predicts the behaviour of the scattered field as a function of jet velocity and tone frequency. Also simulations at other polar angles and a parametric study are presented. These simulations indicate how the 'haystacking' is predicted to vary as a function of the polar angle, and also as a function of the characteristic length, time and convection velocity scales of the turbulence contained in the jet shear layer.
\end{abstract}

\section{Introduction}

Spectral broadening is a phenomenon whereby a tonal sound field interacts with a random time-varying scattering medium, with the result that power is lost from the tone and distributed into a broadband field around the tone frequency. Spectral broadening has been observed in far-field measurements of turbine tones, and to a lesser extent, fan tones radiated from the rear of a turbofan engine. The effect is caused by the interaction of the tones radiated from the engine exhaust duct with the turbulence in the jet shear layers. Turbulent jet shear layers are formed between the hot core jet and cold bypass streams, and also between the bypass and flight streams. Sound radiated from the exhaust propagates through these turbulent shear layers, and owing to the unsteady nature of turbulent flow, this can scatter sound over a range of frequencies. The resulting scattered broadband field, known colloquially as a 'haystack', can be measured well above the jet-noise broadband at some engine conditions.

In the context of this work, when the proportion of scattered energy is small relative to the energy that remains in the tone, this is termed 'weak scattering'. An example of the spectrum from a tonal field which has undergone 'weak' spectral broadening is shown in figure 1, which is taken from the experimental work of Candel, Guedel, \& Julienne. ${ }^{1}$ The level of the tone is around $20 \mathrm{~dB}$ above that of the haystacks. However, spectral broadening can lead to the disappearance of the tone itself, replaced by a broadband hump. In the context of this work, this would be termed 'strong scattering'. 
A detailed analytical model of tone haystacking, in terms of the characteristics of the jet shear layer and the turbulence, has so far been unavailable. In this article, an analytical model is presented and tested against the available experimental results, valid for tones which are weakly scattered as they radiate through a cylindrical jet. This work is based upon a model derived by A.M. Cargill ${ }^{2,3}$ in an internal Rolls-Royce technical report.

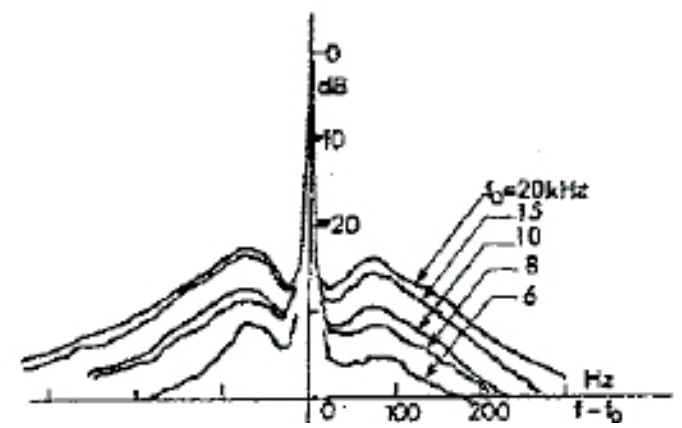

Figure 1. Spectrum of a tone having undergone 'weak' spectral broadening by a jet shear layer, for various incident frequencies $f_{0}$, measured by Candel et al. ${ }^{1}$ (C)1975 IEEE

\section{Previous work}

The scattering of waves by turbulence is of interest to researchers in the fields of astronomy, where atmospheric turbulence presents a problem for ground-based telescopes, and underwater acoustics, where sonar signals are scattered by large-scale turbulence in the oceans. A great deal of work has been done in these areas, but much of it is of limited use in this work, due to large differences in the parameters regimes of interest.

There are limited experimental results on tone haystacking that have been published. Mathews et al. ${ }^{4,5}$ report full-scale engine tests utilized to study core and turbine noise. Their results show the presence of broadband haystacks in the spectrum, centred on the turbine tones. Candel et al. ${ }^{1,6,7}$ report laboratory experiments on model-scale jets. In these experiments a source (approximately a point source) is situated inside a circular or rectangular jet, and a microphone is situated outside. Their measurements provide examples of weak haystacking measured at polar angle equal to $90^{\circ}$. These measurements have been utilized in the present work for validation purposes.

Also there are limited theoretical studies on tone haystacking that have been published. The most relevant published work appears to be by Campos. ${ }^{8} 9$ His model incorporates a number of important effects, including source directivity. The turbulence scattering is modelled by a phase-screen approach; the turbulence is assumed to create a random phase modulation of the field. Mean-flow refraction effects are approximated by modelling the shear layer as an oscillating vortex sheet.

In the context of aeroacoustics, scattering of sound by unsteady flows was reviewed by Cargill. ${ }^{10}$ Subsequently, Cargill developed a theoretical model to predict tone haystacking. An analytical solution for the far-field spectral density due to scattering of sound by a plane turbulent jet is formulated in Refs. [2,3]. This is valid only at polar angles far outside the cone of silence. In this work, following the method proposed by Cargill, the outside cone of silence solution has been derived for a cylindrical jet. Also the method has been extended to determine 
a solution which is valid at all polar angles, including inside the cone of silence. These new analytical solutions are presented in Section III.

An alternative method for modelling the spectral broadening is through numerical simulations. Ewert et al. ${ }^{11}$ describe computational aeroacoustic simulations in which the wave equation for sound in fluids with unsteady inhomogeneous flow is integrated. In this calculation the unsteady turbulent base flow is modelled using a stochastic method to generate turbulence features as provided by time-averaged RANS calculations. The result is a prediction of spectrum shapes which agrees with experiments, but it is noted that this numerical analysis does not take account of scattering between acoustic azimuthal orders: this is accounted for in the current work. Brief details of the analytical model to predict haystacking at polar angles outside the cone of silence, presented in this article in Section III, were included in Ref. [11], which described progress on a DLR/ISVR collaborative research programme on turbine-tone haystacking conducted under the EC TURNEX project.

\section{Methodology}

A typical example of a turbine tone ray path is shown sketched in figure 2 . In this type of 3/4-cowl configuration, the expectation is that most of the scattering occurs in the thicker outer shear layer between the bypass and flight streams. The inner shear layer between the hot core jet and bypass stream is assumed to be thin, since the sound propagates through this layer close to the exit nozzle. In the modelling, the propagation of sound through a single shear layer is considered. Accordingly the turbofan engine's exhaust is modelled by a single-stream cylindrical jet, taking the jet velocity $U_{J}$ equal to the velocity of the bypass stream. Also the fluid outside the jet is assumed to be stationary. This means that the flight stream is omitted in the present analysis.

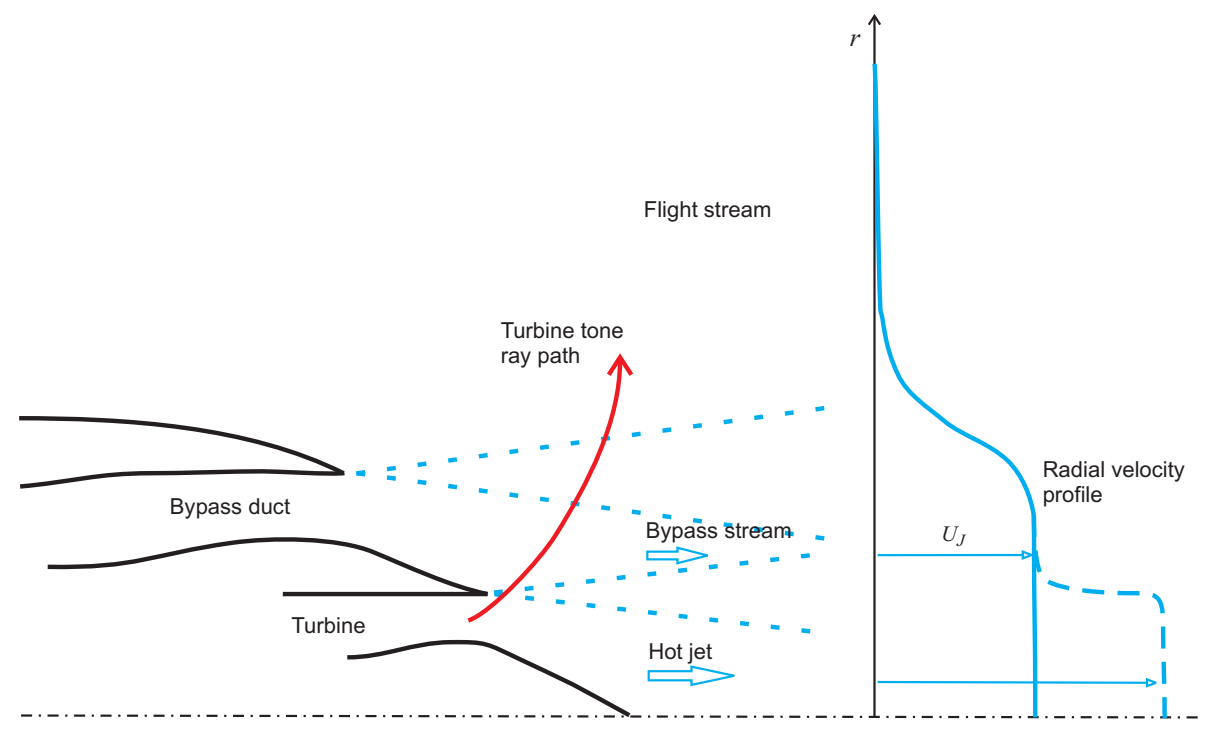

Figure 2. Sketch to illustrate the propagation of a turbine tone through the turbulent jet shear layers formed at the rear of a turbofan engine. The type of radial mean-flow velocity profile used in the modelling is also sketched. Note that the hot jet shown by the dashed line is omitted in the modelling. 


\section{A. Cylindrical jet}

A three-dimensional, parallel, axisymmetric cylindrical jet is considered. Both cylindrical polar coordinates $(x, r, \phi)$ and spherical polar coordinates $(R, \theta, \phi)$ are utilized in the following analysis. These are related to Cartesian coordinates $\left(x_{1}, x_{2}, x_{3}\right)=(x, y, z)$ by

$$
(x, y, z)=(x, r \cos \phi, r \sin \phi)=(R \cos \theta, R \sin \theta \cos \phi, R \sin \theta \sin \phi),
$$

as shown in figure 3 .

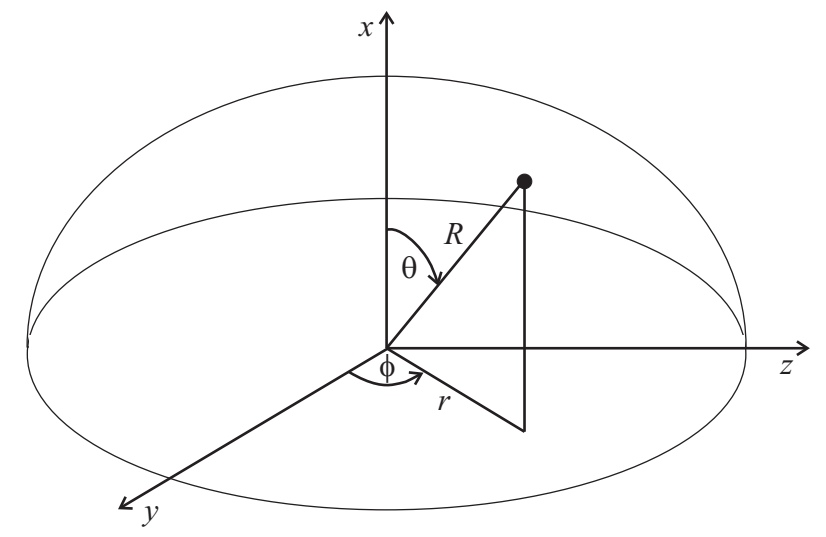

Figure 3. Cylindrical and spherical polar coordinate systems.

The fluid flow is expressed in terms of a steady mean flow plus a small unsteady fluctuating component. Since the mean flow is parallel and axisymmetric, the mean flow pressure, velocity, density and temperature profiles are given by

$$
\begin{aligned}
\bar{p} & =\text { constant } \\
\overline{\mathbf{u}} & =\left(\bar{u}_{x}, \bar{u}_{r}, \bar{u}_{\phi}\right)=(U(r), 0,0) \\
\bar{\rho} & =\bar{\rho}(r) \\
\bar{T} & =\bar{T}(r)
\end{aligned}
$$

respectively. For an isentropic perfect gas absolute temperature and speed of sound are related by $c^{2}=\gamma R T$, where $\gamma=C_{p} / C_{V}$ is the ratio of specific heat capacities, and $R$ is the specific gas constant. Hence (5) is equivalent to

$$
\overline{c^{2}}=\overline{c^{2}}(r) .
$$

The small unsteady fluctuations to the mean flow are then given by $p^{\prime}=p-\bar{p}, \mathbf{u}^{\prime}=\mathbf{u}-\overline{\mathbf{u}}$, $\rho^{\prime}=\rho-\bar{\rho}$ and $c^{2^{\prime}}=c^{2}-\overline{c^{2}}$.

\section{B. Scattering equation}

The scattering of sound by a turbulent flow is modelled using an approximate form of Lilley's equation, a full derivation of which for brevity is omitted here (this will be shown in a future planned article). The scattering equation is expressed in the form

$$
L[p]=S,
$$


where $L$ is the Pridmore-Brown operator, and $S$ denotes the source terms. Using Cartesian tensor notation, in full (7) is

$$
\begin{aligned}
{\left[\frac{\overline{\mathrm{D}}}{\mathrm{D} t}\left(\frac{1}{\overline{c^{2}}} \frac{\overline{\mathrm{D}}^{2}}{\mathrm{D} t^{2}}-\bar{\rho} \nabla \cdot\left(\frac{1}{\bar{\rho}} \nabla\right)\right)+2\left(\frac{\partial \bar{u}_{i}}{\partial x_{j}}\right) \frac{\partial^{2}}{\partial x_{i} \partial x_{j}}\right] p=} & \frac{\overline{\mathrm{D}}}{\mathrm{D} t}\left[\bar{\rho} \frac{\partial}{\partial x_{i}}\left(\frac{F_{i}}{\bar{\rho}}\right)\right]-2\left(\frac{\partial \bar{u}_{i}}{\partial x_{j}}\right) \frac{\partial F_{j}}{\partial x_{i}} \\
& -\bar{\rho} \frac{\overline{\mathrm{D}}^{2} Q}{\mathrm{D} t^{2}}
\end{aligned}
$$

where

$$
\begin{aligned}
F_{i} & \approx \frac{\partial}{\partial x_{k}}\left(\bar{\rho} u_{i}^{\prime} u_{k}^{\prime}\right), \\
Q & \approx \frac{1}{C_{p}} \frac{\partial}{\partial x_{i}}\left(s^{\prime} u_{i}^{\prime}\right),
\end{aligned}
$$

and $s^{\prime}=s-\bar{s}$ denotes the small unsteady fluctuating part of the specific entropy. It is noted that a similar version of equation (8) is derived by Goldstein, ${ }^{12}$ see p. 10, equation (1.24).

For a cylindrical jet, employing cylindrical polar coordinates, it is straightforward to show that the Pridmore-Brown operator is given by

$$
L=\left[\frac{\overline{\mathrm{D}}}{\mathrm{D} t}\left(\frac{1}{\overline{c^{2}}} \frac{\overline{\mathrm{D}}^{2}}{\mathrm{D} t^{2}}-\bar{\rho} \nabla \cdot\left(\frac{1}{\bar{\rho}} \nabla\right)\right)+2\left(\frac{\mathrm{d} U}{\mathrm{~d} r}\right) \frac{\partial^{2}}{\partial x \partial r}\right],
$$

where the mean-flow convected derivative operator

$$
\frac{\overline{\mathrm{D}}}{\mathrm{D} t}=\frac{\partial}{\partial t}+U \frac{\partial}{\partial x} .
$$

It is more difficult to determine the source terms for a cylindrical jet. In the following analysis WKB theory is used, which is a high frequency approximation. As a consequence of this, source terms involving gradients of mean flow quantities are neglected. The remaining sources terms are listed in the appendix - see equation (55).

The solution of the scattering equation, and the ensuing analysis which leads to a closedform expression for the far-field spectral density of a tone 'haystack', is for brevity omitted from this article (this will be shown in a future planned article). In the following section, the solution method for a cylindrical jet is outlined, and the key results are presented.

\section{Outline of solution method}

The pressure perturbation $p^{\prime}$ is described as the sum of two parts, termed the 'incident' and 'scattered' acoustic fields. (In the derivation of (8) pressure perturbations due to the turbulence are assumed to be small and neglected.) The incident field is defined as the field which would be present in the absence of any scattering, i.e. in the absence of turbulence. The scattered field is the field due to the interaction of the turbulence with the incident field. A weak-scattering model is formulated whereby interaction between the scattered field and turbulence is neglected, since the amplitude of the scattering field is assumed to be much less than that of the incident field.

In the following analysis lower-case variables are used to denote incident field quantities and upper-case variables are used to denote scattered field quantities. This necessitates a slight change in notation. The acoustic perturbation is expressed as $p^{\prime}+P^{\prime}$, where now $p^{\prime}$ denotes the incident field and $P^{\prime}$ denotes the scattered field, and $\left|P^{\prime}\right|<<\left|p^{\prime}\right|$ (weak scattering). 
The velocity perturbation $\mathbf{u}^{\prime}$ is described as the sum of a turbulent and acoustic field, i.e. $u_{i}^{\prime}=u_{i t}^{\prime}+u_{i s}^{\prime}$ where subscript $t$ denotes turbulence and subscript $s$ denotes sound. In principle the entropy perturbation $s^{\prime}$ could be expressed in a similar manner, i.e. $s^{\prime}{ }_{t}+s^{\prime}{ }_{s}$, but the acoustic entropy fluctuations are assumed to be small and neglected. In the source terms $F_{i}(9)$ and $Q$ (10) only turbulent/acoustic field interactions are retained, so

$$
\begin{aligned}
u_{i}^{\prime} u_{j}^{\prime} & \approx u_{i t}^{\prime} u_{j_{s}}^{\prime}+u_{i s}^{\prime} u_{j_{t}}^{\prime}, \\
s^{\prime} u_{i}^{\prime} & \approx s_{t}^{\prime} u_{i s}^{\prime} .
\end{aligned}
$$

The governing equations are solved by taking Fourier transforms in $x$ and $t$ and a Fourier series in $\phi$. Denoting transformed variables by a tilde, the incident field is found by solving

$$
\widetilde{L}\left[\widetilde{p^{\prime}}\right]=0,
$$

and the scattered field is found by solving

$$
\widetilde{L}\left[\widetilde{P^{\prime}}\right]=\widetilde{S}
$$

where the terms in $\widetilde{S}$ are functions of the transformed turbulent/acoustic field interactions (13) and (14). Following the weak scattering assumption, in the source terms $u_{i s}^{\prime}$ is taken to be the incident acoustic field, and can be expressed in terms of $p^{\prime}$ (via the linearised momentum equation). This allows the solution of (16) to be formulated in terms of a Green function integral equation, i.e.

$$
{\widetilde{P^{\prime}}}_{M}(K, r, \Omega)=\int_{r_{0}=0}^{\infty} \widetilde{G}_{M}\left(r \mid r_{0}\right) \widetilde{S}_{M}\left(r_{0}\right) r_{0} \mathrm{~d} r_{0},
$$

where $K, M$ and $\Omega$ denote the axial wavenumber, azimuthal order, and frequency respectively of the scattered acoustic field. An approximate Green function which is valid in the high-frequency limit is derived by utilizing the WKB-method in conjunction with a Langer transformation. (Similar approximate high-frequency Green functions of Lilley's equation have been derived previously, for example see Wundrow \& Khavaran. ${ }^{13}$ ) In (17) the scattered acoustic field is expressed as the integral over all radial source positions of the product of the transformed Green function multiplied by the transformed source terms in (16). In practice this integral will be evaluated only at radial locations within the jet shear layer, since the turbulence is confined to this region. A separate calculation is performed for each different value of the scattered azimuthal order $M$. The source terms contain products of velocity and entropy fluctuations. Having separated the velocity and entropy fluctuations into their acoustic and non-acoustic (turbulent) components, cf. (13) and (14), the acoustic component of the source terms is found by solving (15) to find the incident acoustic field. Modelling the source terms in this manner is reasonable only provided that the scattering is sufficiently weak. The incident acoustic field is found in the same manner as the Green function, and again is valid only in the high-frequency limit.

Having obtained this high-frequency solution of the scattering equation, $\widetilde{P}^{\prime} M$, the spectral density $\widetilde{P}_{M}$ is formed by taking

$$
\widetilde{P}_{M}=\left\langle{\widetilde{P^{\prime}}}_{M}{\widetilde{P^{\prime}}}_{M}^{\star}\right\rangle,
$$

where $\langle.$.$\rangle denotes an ensemble average, and * denotes the complex conjugate. This is convenient$ because the description of the turbulence is now required in terms of turbulence correlation functions and spectra, for which there are a number of models which may be utilized. Since the mean flow is steady, parallel, and axisymmetric, it is assumed that the turbulence is statistically 
stationary in the variables $x, \phi$ and $t$. Then the turbulent velocity and entropy cross-correlation functions

$$
\begin{aligned}
R_{i j} & =\left\langle u_{i t}^{\prime}\left(x_{1}, r_{01}, \phi_{1}, t_{1}\right) u_{j}^{\prime \star}\left(x_{2}, r_{02}, \phi_{2}, t_{2}\right)\right\rangle, \\
R_{s} & =\left\langle s_{t}^{\prime}\left(x_{1}, r_{01}, \phi_{1}, t_{1}\right) s_{t}^{\prime \star}\left(x_{2}, r_{02}, \phi_{2}, t_{2}\right)\right\rangle,
\end{aligned}
$$

respectively, are functions of only

$$
\mu_{x}=x_{1}-x_{2}, \quad \mu_{r}=r_{01}-r_{02}, \quad \mu_{\phi}=\phi_{1}-\phi_{2}, \quad \mu_{t}=t_{1}-t_{2} \text { and } \bar{r}=\frac{1}{2}\left(r_{01}+r_{02}\right),
$$

where $\mu$ denotes a source separation variable, and $\bar{r}$ is the average radial source position. Also it is assumed that the turbulent velocity and entropy fluctuations are uncorrelated. Accordingly the turbulent velocity and entropy cross-spectra are given by

$$
\begin{array}{r}
\Phi_{i j}\left(K-k, \bar{r}, \Gamma_{M}-\gamma_{m}, M-m, \Omega-\omega\right)=\int_{\mu_{x}=-\infty}^{\infty} \int_{\mu_{r}=-\infty}^{\infty} \int_{\mu_{\phi}=-\pi}^{\pi} \int_{\mu_{t}=-\infty}^{\infty} R_{i j} \times \\
e^{\mathrm{i}(K-k) \mu_{x}} e^{\mathrm{i}\left(\Gamma_{M}-\gamma_{m}\right) \mu_{r}} e^{\mathrm{i}(M-m) \mu_{\phi}} e^{-\mathrm{i}(\Omega-\omega) \mu_{t}} \mathrm{~d} \mu_{t} \mathrm{~d} \mu_{\phi} \mathrm{d} \mu_{r} \mathrm{~d} \mu_{x}, \\
S\left(K-k, \bar{r}, \Gamma_{M}-\gamma_{m}, M-m, \Omega-\omega\right)=\int_{\mu_{x}=-\infty}^{\infty} \int_{\mu_{r}=-\infty}^{\infty} \int_{\mu_{\phi}=-\pi}^{\pi} \int_{\mu_{t}=-\infty}^{\infty} R_{s} \times \\
e^{\mathrm{i}(K-k) \mu_{x}} e^{\mathrm{i}\left(\Gamma_{M}-\gamma_{m}\right) \mu_{r}} e^{\mathrm{i}(M-m) \mu_{\phi}} e^{-\mathrm{i}(\Omega-\omega) \mu_{t}} \mathrm{~d} \mu_{t} \mathrm{~d} \mu_{\phi} \mathrm{d} \mu_{r} \mathrm{~d} \mu_{x},
\end{array}
$$

respectively. Note that $k, m$ and $\omega$ denote the axial wavenumber, azimuthal order, and frequency respectively of the incident acoustic field. Also $\Gamma_{M}$ and $\gamma_{m}$ are effective radial wavenumbers of the scattered and incident acoustic field respectively.

Further simplifications to $\widetilde{P}_{M}$ are made by taking the far-field limit as $r \rightarrow \infty$, and by assuming that the turbulence correlation lengthscale is small. In practice the turbulent velocity and entropy cross-correlation functions are required to be negligible unless $\mu_{r}$ is sufficiently small. It is assumed that $\mu_{r}$ is comparable with the turbulence correlation lengthscale, and is typically less than a tenth of the jet diameter. Under these assumptions, the far-field spectral density is given by

$$
\widetilde{P}_{M}(K, r, \Omega) \approx \frac{1}{(2 \pi)^{3}} \sum_{m=-\infty}^{\infty} \int_{\omega=-\infty}^{\infty} \int_{k=-\infty}^{\infty}\left|\frac{\tilde{p}_{m_{\infty}}^{\prime}}{\sqrt{r}}\right|^{2} \mathcal{H}(K, M, \Omega, k, m, \omega) \mathrm{d} \omega \mathrm{d} k,
$$

where $\mathcal{H}(K, M, \Omega, k, m, \omega)$ is given by an integral over the source region:

$$
\begin{aligned}
\mathcal{H}(K, M, \Omega, k, m, \omega)= & \int_{\bar{r}=0}^{\infty} \frac{8 \pi^{2}}{\bar{D}^{2}}\left(\frac{D_{\infty}}{d_{\infty}}\right)^{2}\left(\frac{\gamma_{m_{\infty}}}{\Gamma_{M \infty}}\right)\left(\frac{\bar{\xi}_{m}}{\bar{\gamma}_{m}^{2}}\right)^{\frac{1}{2}}\left(\frac{\bar{\zeta}_{M}}{\bar{\Gamma}_{M}^{2}}\right)^{\frac{1}{2}} \times \\
& {\left[\operatorname{Bi}^{2}\left(-\bar{\xi}_{m}\right)+\operatorname{Ai}^{2}\left(-\bar{\xi}_{m}\right)\right] \mathrm{Ai}^{2}\left(-\bar{\zeta}_{M}\right) \times } \\
& {\left[|\bar{\Lambda}|^{2} K^{2} \Phi_{x x}+\bar{\Lambda} \bar{\Delta}^{\star} K \bar{\Gamma}_{M}^{\star} \Phi_{x r}+|\bar{\Lambda}|^{2} K(M / \bar{r}) \Phi_{x \phi}\right.} \\
& +\bar{\Lambda}^{\star} \bar{\Delta} \bar{\Gamma}_{M} K \Phi_{r x}+|\bar{\Delta}|^{2}\left|\bar{\Gamma}_{M}\right|^{2} \Phi_{r r}+\bar{\Lambda}^{\star} \bar{\Delta}_{M}(M / \bar{r}) \Phi_{r \phi} \\
& +|\bar{\Lambda}|^{2}(M / \bar{r}) K \Phi_{\phi x}+\bar{\Lambda} \bar{\Delta}^{\star}(M / \bar{r}) \bar{\Gamma}_{M}^{\star} \Phi_{\phi r}+|\bar{\Lambda}|^{2}(M / \bar{r})^{2} \Phi_{\phi \phi} \\
& \left.+|\bar{\Lambda}|^{2} \frac{\bar{D}^{2}}{4 C_{p}^{2}} S\right] \mathrm{d} \bar{r}
\end{aligned}
$$


In (24) the term ${\widetilde{p^{\prime}}}_{m_{\infty}} / \sqrt{r}$ is the amplitude of the transformed far-field incident pressure field. In (25) a bar denotes a quantity evaluated at $r=\bar{r}$, whilst a subscript $\infty$ denotes a quantity evaluated as $r \rightarrow \infty$. The turbulent velocity and entropy cross-spectra $\Phi$ and $S$ are evaluated at the difference wavenumbers and frequencies $\left(K-k, \bar{r}, \Gamma_{M}-\gamma_{m}, \quad M-m, \Omega-\omega\right)$ taking $\Gamma_{M}-$ $\gamma_{m}=0$. Setting $\Gamma_{M}=\gamma_{m}$ when evaluating $\Phi$ and $S$ in (25) is a consequence that follows from assuming that $\mu_{r}$ is small when deriving the function $\mathcal{H}$. Also the following variables in (25) are defined as follows. Doppler factors $d$ and $D$ are

$$
d=\omega-k U \quad \text { and } \quad D=\Omega-K U .
$$

The effective radial wavenumbers $\gamma_{m}$ and $\Gamma_{M}$ are given by

$$
\gamma_{m}^{2}=\frac{d^{2}}{\overline{c^{2}}}-k^{2}-\frac{m^{2}}{r^{2}} \quad \text { and } \quad \Gamma_{M}^{2}=\frac{D^{2}}{\overline{c^{2}}}-K^{2}-\frac{M^{2}}{r^{2}} .
$$

The turning points $r_{m_{c}}$ and $R_{M_{c}}$ are where

$$
\gamma_{m}^{2}\left(r_{m_{c}}\right)=0 \quad \text { and } \quad \Gamma_{M}^{2}\left(R_{M_{c}}\right)=0 .
$$

The variables $\xi_{m}$ and $\zeta_{M}$ are given by

$$
\begin{gathered}
\xi_{m}=\left(\frac{3}{2} \int_{r_{m c}}^{r} \gamma_{m}\left(r^{\prime}\right) \mathrm{d} r^{\prime}\right)^{\frac{2}{3}}, r>r_{m c} \quad \text { and } \xi_{m}=-\left(\frac{3}{2} \int_{r}^{r_{m c}}\left|\gamma_{m}\left(r^{\prime}\right)\right| \mathrm{d} r^{\prime}\right)^{\frac{2}{3}}, r<r_{m_{c}}, \\
\zeta_{M}=\left(\frac{3}{2} \int_{R_{M c}}^{r} \Gamma_{M}\left(r^{\prime}\right) \mathrm{d} r^{\prime}\right)^{\frac{2}{3}}, r>R_{M c} \quad \text { and } \zeta_{M}=-\left(\frac{3}{2} \int_{r}^{R_{M c}}\left|\Gamma_{M}\left(r^{\prime}\right)\right| \mathrm{d} r^{\prime}\right)^{\frac{2}{3}}, r<R_{M c},
\end{gathered}
$$

where $r^{\prime}$ is a dummy integration variable. The functions $\psi$ and $\Psi$ are defined as

$$
\begin{aligned}
& \psi(x)=-\mathrm{i}(-x)^{-\frac{1}{2}} \frac{\left[\mathrm{Ai}(x) \mathrm{Ai}^{\prime}(x)+\mathrm{Bi}(x) \mathrm{Bi}^{\prime}(x)+\mathrm{i} / \pi\right]}{\mathrm{Bi}^{2}(x)+\mathrm{Ai}^{2}(x)}, \\
& \Psi(x)=\mathrm{i}(-x)^{-\frac{1}{2}} \frac{\mathrm{Ai}^{\prime}(x)}{\mathrm{Ai}(x)}
\end{aligned}
$$

where $\mathrm{Ai}$ and $\mathrm{Bi}$ are Airy functions. Finally, the functions $\Lambda$ and $\Delta$ are defined as

$$
\begin{aligned}
& \Lambda(r)=K k+\Gamma_{M} \gamma_{m} \Psi \psi+\frac{M m}{r^{2}} \\
& \Delta(r)=K k \Psi+\Gamma_{M} \gamma_{m} \psi+\frac{M m \Psi}{r^{2}}
\end{aligned}
$$

Equation (24) gives the far-field spectral density of the scattered field, in terms of the wavenumber $K$ and cylindrical radius $r$. In general however, the evaluation of the spectrum at a given polar angle $\Theta$ at a spherical distance $R$ from the source will be of more interest. Thus the far-field scattered acoustic field $\widetilde{P^{\prime}}{ }_{M}(K, r, \Omega)$ is transformed to a function $\widetilde{P}_{M}{ }_{M}(R, \Theta, \Omega)$. This requires the inverse Fourier transform in $k$ to be evaluated, which in the limit as $R \rightarrow \infty$ can be determined using the the method of stationary phase. This transforms the spectral density in terms of a wavenumber spectrum to a mean-square pressure directivity function. The final result 
is expressed in terms of an integral over polar angle of the mean-square pressure directivity of the incident acoustic field $\widetilde{p}_{m_{d}}$ multiplied by the function $\mathcal{H}(25)$, which gives the mean-square pressure directivity of the scattered acoustic field $\widetilde{P}_{M_{D}}$ :

$$
\begin{aligned}
\widetilde{P}_{M_{D}}(\Theta, \Omega) \approx & \left|K_{\infty}\right| \sin \theta \frac{1}{(2 \pi)^{3}} \sum_{m=-\infty}^{\infty} \int_{\omega=-\infty}^{\infty} \int_{\theta=0}^{\pi} \widetilde{p}_{m_{d}}(\theta, \omega) \times \\
& \mathcal{H}\left(\left|K_{\infty}\right| \cos \Theta, M, \Omega,\left|k_{\infty}\right| \cos \theta, m, \omega\right) \mathrm{d} \omega \mathrm{d} \theta .
\end{aligned}
$$

Equation (35) is the key result of this work. The far-field mean-square pressure directivities of the incident and scattered fields are linked via the transfer function $\mathcal{H}(25)$. It is emphasized that this result is valid at all polar angles $\Theta$ in the range $(0, \pi)$, which includes observer angles inside the cone of silence.

In the original work by Cargill, ${ }^{3}$ a simpler form of the function $\mathcal{H}$ is derived which is valid only at polar angles far outside the cone of silence. This is obtained by making several additional assumptions. The main additional assumption is that the source location $r_{0}$ is always located above any turning points, i.e. $r_{0}>r_{m_{c}}$ and $r_{0}>r_{M_{c}}$. This permits the Airy functions Ai and $\mathrm{Bi}$ to be expressed in terms of their large argument asymptotic limits. Also reflections at the turning point $r_{M_{c}}$ are ignored which permits a simpler version of the Green function to be utilized. Then at polar angles outside the cone of silence, following the solution method proposed by Cargill, an approximate version of $\mathcal{H}$ is given by

$$
\begin{aligned}
\mathcal{H}(K, M, \Omega, k, m, \omega) \approx & \int_{\bar{r}=0}^{\infty} \frac{2}{\bar{D}^{2}}\left(\frac{D_{\infty}}{d_{\infty}}\right)^{2}\left(\frac{\gamma_{m_{\infty}}}{\Gamma_{M_{\infty}}}\right)\left(\frac{1}{\bar{\gamma}_{m}^{2}}\right)^{\frac{1}{2}}\left(\frac{1}{\bar{\Gamma}_{M}^{2}}\right)^{\frac{1}{2}} \times \\
& \left(K k+\bar{\Gamma}_{M} \bar{\gamma}_{m}+\frac{M m}{\bar{r}^{2}}\right)^{2} \times \\
& {\left[K^{2} \Phi_{x x}+K \bar{\Gamma}_{M} \Phi_{x r}+K(M / \bar{r}) \Phi_{x \phi}\right.} \\
& +\bar{\Gamma}_{M} K \Phi_{r x}+\bar{\Gamma}_{M}^{2} \Phi_{r r}+\bar{\Gamma}_{M}(M / \bar{r}) \Phi_{r \phi} \\
& +(M / \bar{r}) K \Phi_{\phi x}+(M / \bar{r}) \bar{\Gamma}_{M} \Phi_{\phi r}+(M / \bar{r})^{2} \Phi_{\phi \phi} \\
& \left.+\frac{\bar{D}^{2}}{4 C_{p}^{2}} S\right] \mathrm{d} \bar{r}
\end{aligned}
$$

In (36) $\Phi$ and $S$ are evaluated at the difference wavenumbers and frequencies $\left(K-k, \bar{r}, \Gamma_{M}-\gamma_{m}\right.$, $M-m, \Omega-\omega)$. Unlike in (25) it is not necessary to set $\Gamma_{M}=\gamma_{m}$ when evaluating $\Phi$ and $S$. This is because the procedure to derive the approximate version of $\mathcal{H}(36)$ is rather different to the derivation of (25). However, since $\mu_{r}$ is assumed to be small, then setting $\Gamma_{M}=\gamma_{m}$ or not when evaluating $\Phi$ and $S$ has minimal effect in either case.

At polar angles far outside the cone of silence, $\widetilde{P}_{M_{D}}(35)$ may be calculated with $\mathcal{H}$ given by (25) or (36). It is demonstrated in Section IV that at $\Theta=90^{\circ}$ results using either transfer function are found to be very similar, but computing $\mathcal{H}$ using (36) is significantly faster than using (25). The function $\mathcal{H}$ is evaluated by an integration over the average source position $\bar{r}=\left(r_{01}+r_{02}\right) / 2$, where the two source locations are $r_{01}$ and $r_{02}$. It is assumed that if $r_{01}$ and/or $r_{02}$ are located sufficiently far from the turbulent shear layer, then the turbulent velocity and entropy cross-correlations will be negligible. This means that the integration over $\bar{r}$ in (25) or (36) in practice is evaluated only over a finite range which spans the shear layer. 


\section{Results}

Equation (35) expresses the far-field mean-square pressure directivity of the scattered field, $\widetilde{P}_{M_{D}}$, in terms of the far-field mean-square pressure directivity of the incident field, $\widetilde{p}_{m_{d}}$. This requires $\widetilde{p}_{m_{d}}$ multiplied by a transfer function $\mathcal{H}$ to be integrated over the incident frequency $\omega$ and over the range of incident angles $\theta$, then summed over all azimuthal orders $m$ of the incident field. However, in each of the computed haystacks presented in this article the scattered field has been calculated for a single frequency, single azimuthal order incident field. This means that the integration over $\omega$ and summation over $m$ in (35) are not required, which significantly alleviates the computational cost to calculate the scattered field.

\section{A. Experimental measurements by Candel et al.}

There are few published examples of experimental measurements of tone haystacking, and of the results available, the most suitable for validation of the current model is the data in Candel et al. ${ }^{1,6}$ In Ref. [1], the authors describe experimental tests conducted at the von Karman Institute in a $3 \mathrm{~m}$ diameter open jet wind tunnel. A sketch of the experimental setup is shown in figure 4. A monochromatic sound source was placed at the centreline of the jet. The radiated sound waves pass through the turbulent shear layer, formed between the cylindrical jet and quiescent air outside the jet, to reach a microphone placed outside the jet. The microphone was positioned at a polar angle of $90^{\circ}$ from the source. The axial location of the source and microphone could be changed. Measured results are reproduced in this article that were obtained at axial stations $0.5 \mathrm{~m}$ and $1.95 \mathrm{~m}$ from the jet nozzle. The thickness of the shear layer is appreciably wider at the latter axial station. The haystacked field was measured for a range of source frequencies from $4 \mathrm{kHz}$ to $20 \mathrm{kHz}$, and the jet velocity was varied from $20 \mathrm{~ms}^{-1}$ to $60 \mathrm{~ms}^{-1}$.

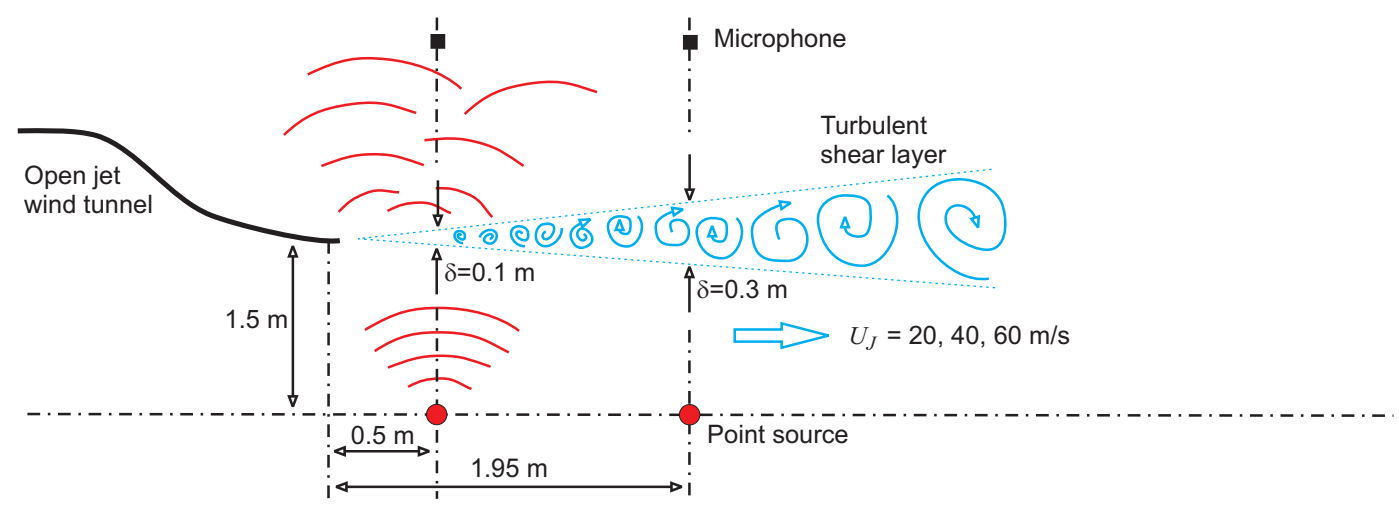

Figure 4. Sketch of experimental set-up used by Candel et al. A point source is placed on the centreline of a jet, and a microphone placed outside the jet at a polar angle approximately $90^{\circ}$ from the source.

In Ref. [1] it is shown that the shear layer velocity profile $U(r)$ is approximately given by

$$
\frac{U}{U_{J}}=\frac{1}{2}(1-\tanh \eta), \quad \eta=\frac{2}{\delta}\left(r-r_{c}\right)
$$

where $U_{J}$ is the velocity of the jet, $\delta$ is the thickness of the shear layer, and $r_{c}$ is the jet radius. Also in Ref. [6] estimates of the shear layer thickness and jet radius are given by

$$
\delta=0.17 x
$$




$$
r_{c}=1.47+0.048 x
$$

where $x$ is the distance in metres downstream of the nozzle.

Measurements of the far-field radiation pattern are shown to be fairly omnidirectional, so in the simulations a uniform directivity is assumed at all polar angles $\theta>\theta_{c}$, where the critical angle $\theta_{c}$ is given by

$$
\cos \theta_{c}=\frac{c_{\infty}}{c_{J}+U_{J}} .
$$

Since the jet is unheated, it is assumed that $c_{J}=c_{\infty}$. The critical angles for the three jet velocities used in the experiments by Candel et al. are:

\begin{tabular}{cc}
$U_{J}$ & $\theta_{c}$ \\
\hline $20 \mathrm{~ms}^{-1}$ & $19.2^{\circ}$ \\
$40 \mathrm{~ms}^{-1}$ & $26.5^{\circ}$ \\
$60 \mathrm{~ms}^{-1}$ & $31.8^{\circ}$
\end{tabular}

Table 1. Critical angle $\theta_{c}$.

Also since the jet is unheated, the turbulent velocity fluctuations are assumed to be the primary scattering mechanism, so in the simulations the turbulent entropy fluctuations are neglected. The turbulent velocity spectrum $\Phi_{i j}$ is found from a model we refer to as a GaussianHIT correlation, since theory related to Homogeneous Isotropic Turbulence is used to derive this function. The turbulence is assumed isotropic and axially homogeneous, and confined within the shear layer. The turbulence correlation lengthscale $l$ is assumed to be small compared to the jet radius. Simulations are calculated for both frozen and non-frozen turbulence models. In the former, it is assumed that the turbulence convects downstream at velocity $U_{c}$, but is otherwise time-independent. In the latter, the turbulence correlation timescale $\tau$ is included as an additional parameter in the model.

\section{B. Gaussian-HIT correlation}

Though the Gaussian correlation function is commonly used to describe turbulent flows, an alternative formulation is considered in this work. Batchelor ${ }^{14}$ has shown that in homogeneous isotropic turbulence, the longitudinal and lateral velocity correlations are related to one another in a certain manner. This concept has been discussed more recently by Ewert. ${ }^{15}$ Through consideration of this relationship, a Gaussian-HIT (Homogeneous Isotropic Turbulence) correlation function can be derived. Assuming frozen turbulence, this correlation function is

$$
\begin{aligned}
& R_{x x}=\overline{u_{t x}^{\prime} u_{t x}^{\prime}}(\bar{r})\left(1-\frac{\mu_{r}^{2}}{l^{2}}\right)\left(1-\frac{\bar{r}^{2} \mu_{\phi}^{2}}{l^{2}}\right) \exp \left\{-\left(\frac{\left(\mu_{x}-U_{c} \mu_{t}\right)^{2}+\mu_{r}^{2}+\bar{r}^{2} \mu_{\phi}^{2}}{l^{2}}\right)\right\} \\
& R_{r r}=\overline{u_{t r}^{\prime} u_{t r}^{\prime}}(\bar{r})\left(1-\frac{\left(\mu_{x}-U_{c} \mu_{t}\right)^{2}}{l^{2}}\right)\left(1-\frac{\bar{r}^{2} \mu_{\phi}^{2}}{l^{2}}\right) \exp \left\{-\left(\frac{\left(\mu_{x}-U_{c} \mu_{t}\right)^{2}+\mu_{r}^{2}+\bar{r}^{2} \mu_{\phi}^{2}}{l^{2}}\right)\right\} \\
& R_{\phi \phi}=\overline{u_{t \phi}^{\prime} u_{t \phi}^{\prime}}(\bar{r})\left(1-\frac{\left(\mu_{x}-U_{c} \mu_{t}\right)^{2}}{l^{2}}\right)\left(1-\frac{\mu_{r}^{2}}{l^{2}}\right) \exp \left\{-\left(\frac{\left(\mu_{x}-U_{c} \mu_{t}\right)^{2}+\mu_{r}^{2}+\bar{r}^{2} \mu_{\phi}^{2}}{l^{2}}\right)\right\}
\end{aligned}
$$

The quantity $\overline{u_{t i}^{\prime} u_{t j}^{\prime}}$ is the turbulence intensity. It is assumed that $\overline{u_{t i}^{\prime} u_{t j}^{\prime}}=0$ for $i \neq j$; measured values for $i=j$ are given by Candel et al. ${ }^{1}$ 
The inclusion of the factor $\exp \left\{-\left|\mu_{t}\right| / \tau\right\}$ in the correlation functions $R_{x x}, R_{r r}$ and $R_{\phi \phi}$ can be used to describe non-frozen turbulence. This temporal exponential term has been proposed by Morris \& Boluriaan. ${ }^{16}$

The cross-spectra are then given by a Fourier transform as

$$
\begin{aligned}
\Phi_{x x}= & \frac{\overline{u_{t x}^{\prime} u_{t x}^{\prime}}(\bar{r})}{4 \bar{r}} l^{3} \pi^{\frac{3}{2}} \Pi\left(1+\frac{l^{2}(\Gamma-\gamma)^{2}}{2}\right)\left(1+\frac{l^{2}(M-m)^{2}}{2 \bar{r}^{2}}\right) \times \\
& \exp \left\{-\frac{l^{2}}{4}\left((K-k)^{2}+(\Gamma-\gamma)^{2}+\frac{(M-m)^{2}}{\bar{r}^{2}}\right)\right\}, \\
\Phi_{r r}= & \frac{\overline{u_{t r}^{\prime} u_{t r}^{\prime}}(\bar{r})}{4 \bar{r}} l^{3} \pi^{\frac{3}{2}} \Pi\left(1+\frac{l^{2}(K-k)^{2}}{2}\right)\left(1+\frac{l^{2}(M-m)^{2}}{2 \bar{r}^{2}}\right) \times \\
& \exp \left\{-\frac{l^{2}}{4}\left((K-k)^{2}+(\Gamma-\gamma)^{2}+\frac{(M-m)^{2}}{\bar{r}^{2}}\right)\right\}, \\
\Phi_{\phi \phi}= & \frac{u_{t \phi}^{\prime} u_{t \phi}^{\prime}(\bar{r})}{4 \bar{r}} l^{3} \pi^{\frac{3}{2}} \Pi\left(1+\frac{l^{2}(K-k)^{2}}{2}\right)\left(1+\frac{l^{2}(\Gamma-\gamma)^{2}}{2}\right) \times \\
& \exp \left\{-\frac{l^{2}}{4}\left((K-k)^{2}+(\Gamma-\gamma)^{2}+\frac{(M-m)^{2}}{\bar{r}^{2}}\right)\right\},
\end{aligned}
$$

where, in the case of frozen turbulence

$$
\Pi=2 \pi \delta\left((\Omega-\omega)-(K-k) U_{c}\right),
$$

or, in the case of non-frozen turbulence

$$
\Pi=\frac{2 \tau}{1+\tau^{2}\left[(\Omega-\omega)-(K-k) U_{c}\right]^{2}} .
$$

\section{Comparison with experiment}

Given the spectra (44)-(46), the far-field mean-square pressure directivity $\widetilde{P}_{M_{D}}$ (35) with the approximate (outside cone of silence) function $\mathcal{H}$ given by (36) has been evaluated for the cases considered in Candel et al. ${ }^{1}$ It is emphasized that this validation is restricted in scope because all the haystacking measurements in Ref. [1] were obtained at polar angle $\Theta=90^{\circ}$. In this article some additional simulations at $\Theta<90^{\circ}$ are also presented but there is no measured data available for comparison.

Measured values from the experiment are used for most of the required parameters, apart from the turbulence correlation length and timescales. Candel et al. ${ }^{1}$ propose the following estimates: $U_{c}=0.5 U_{J}$ and $l \approx 3.2 \delta$. There is no estimate in Ref. [1] of $\tau$. The measured haystacks exhibit a 'double-humped' structure. Candel et al. propose that the peaks in the lateral sidebands of the measured haystacks occur with frequency shift given by

$$
\left|\Delta_{ \pm}\right| \approx \frac{U_{c}}{l}
$$

In this work, the turbulent velocity cross-spectra have a 'double-humped' structure, since they contain terms of the form

$$
f(x)=\left(1+\frac{x^{2}}{2}\right) e^{-x^{2} / 4} .
$$


The turning points of the function $f(x)$ are at $x=-\sqrt{2}, 0$ and $\sqrt{2} ; \pm \sqrt{2}$ are local maximum points. At $\Theta=90^{\circ}$ the axial wavenumber of the scattered field $K=\left|K_{\infty}\right| \cos \Theta=0$, so the term $K^{2} \Phi_{x x}$ in (36) will equal zero. Comparing (50) with (45) and (46), $\bar{\Gamma}_{M}^{2} \Phi_{r r}$ and $(M / \bar{r})^{2} \Phi_{\phi \phi}$ in (36) both contain the term $f(x)$ where $x=l(K-k)$. It follows that local maxima occur at

$$
l(K-k)= \pm \sqrt{2} .
$$

In the case of frozen turbulence $\Phi_{i i}$ is non-zero only if

$$
(\Omega-\omega)-(K-k) U_{c}=0,
$$

following (47). Combining (51) and (52), an estimate of the frequency shift $\Delta_{ \pm}$is given by

$$
\begin{aligned}
& \left(\frac{\Omega-\omega}{U_{c}}\right) l & = \pm \sqrt{2}, \\
\Rightarrow \quad & \left(\frac{2 \pi \Delta_{ \pm}}{U_{c}}\right) l & = \pm \sqrt{2}, \\
\Rightarrow \quad & \left|\Delta_{ \pm}\right| & =\frac{\sqrt{2}}{2 \pi} \frac{U_{c}}{l} .
\end{aligned}
$$

Using (49), Candel et al. ${ }^{1}$ estimate that $l \approx 3.2 \delta$, which seems rather large. However, using (54) instead of $(49)$ gives $l \approx 0.72 \delta$. Owing to uncertainty regarding the appropriate value of $l$, in this work it is assumed that the turbulence correlation length scale is comparable with the thickness of the shear layer, i.e. $l \approx \delta$. This is consistent with the findings in Ref. [1] that it is the large-scale coherent structures that affect the scattered sound field, and also this is more consistent with other examples of turbulence measurements in a shear layer, for example see Harper-Bourne. ${ }^{17}$ Also in this work it is assumed that $\tau U_{c} / l \approx 4$.

\section{1. $\Theta=90^{\circ}$}

Results are shown in figures 5 and 6 using the frozen and non-frozen turbulence models respectively. The simulations are for a sum of scattered azimuthal orders $M=-75$ to $M=75$, and have been normalized so that the tone level is $0 \mathrm{~dB}$. The measurements from Candel et al. ${ }^{1}$ were normalized in a similar manner.

In these results, the 'double-humped' shape of the spectra are reproduced: this is due to the fact that the turbulent velocity cross-spectra (44)-(46) are themselves of a 'double-humped' form. There is little difference between the predictions with frozen or non-frozen turbulence, apart from the haystacks are slightly more concave at large separation frequencies $F-f$ using the non-frozen turbulence model. This better matches the shape of the measured haystacks.

In varying frequency or velocity, the model correctly predicts the trends in the behaviour of the scattered field. For example, the relative levels between predictions, for different frequencies or jet velocities, are correctly predicted. Also, the location of the peaks in the lateral sidebands, for different frequencies or jet velocities, are correctly predicted as well.

However, notably at $x=0.5 \mathrm{~m}$, the absolute levels of the predicted haystacks are less than the measured levels. A small parametric study is included later in this section to investigate how the absolute levels of the predicted haystacks are affected by the different parameters in the model. 
(a)

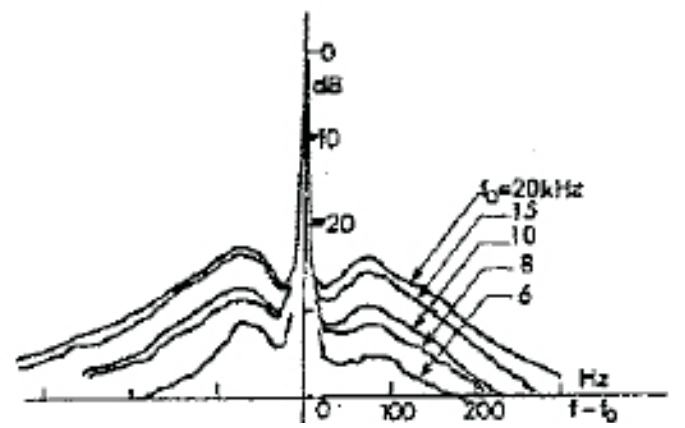

(c)

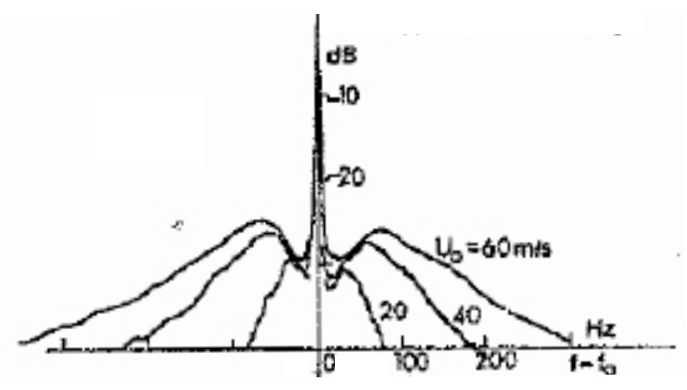

(d)

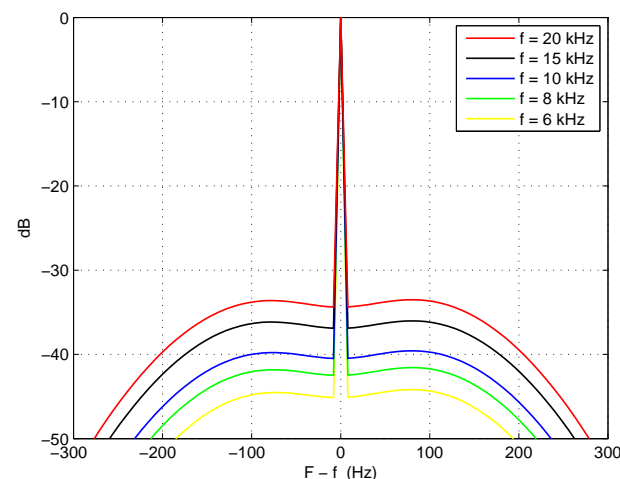

(b)
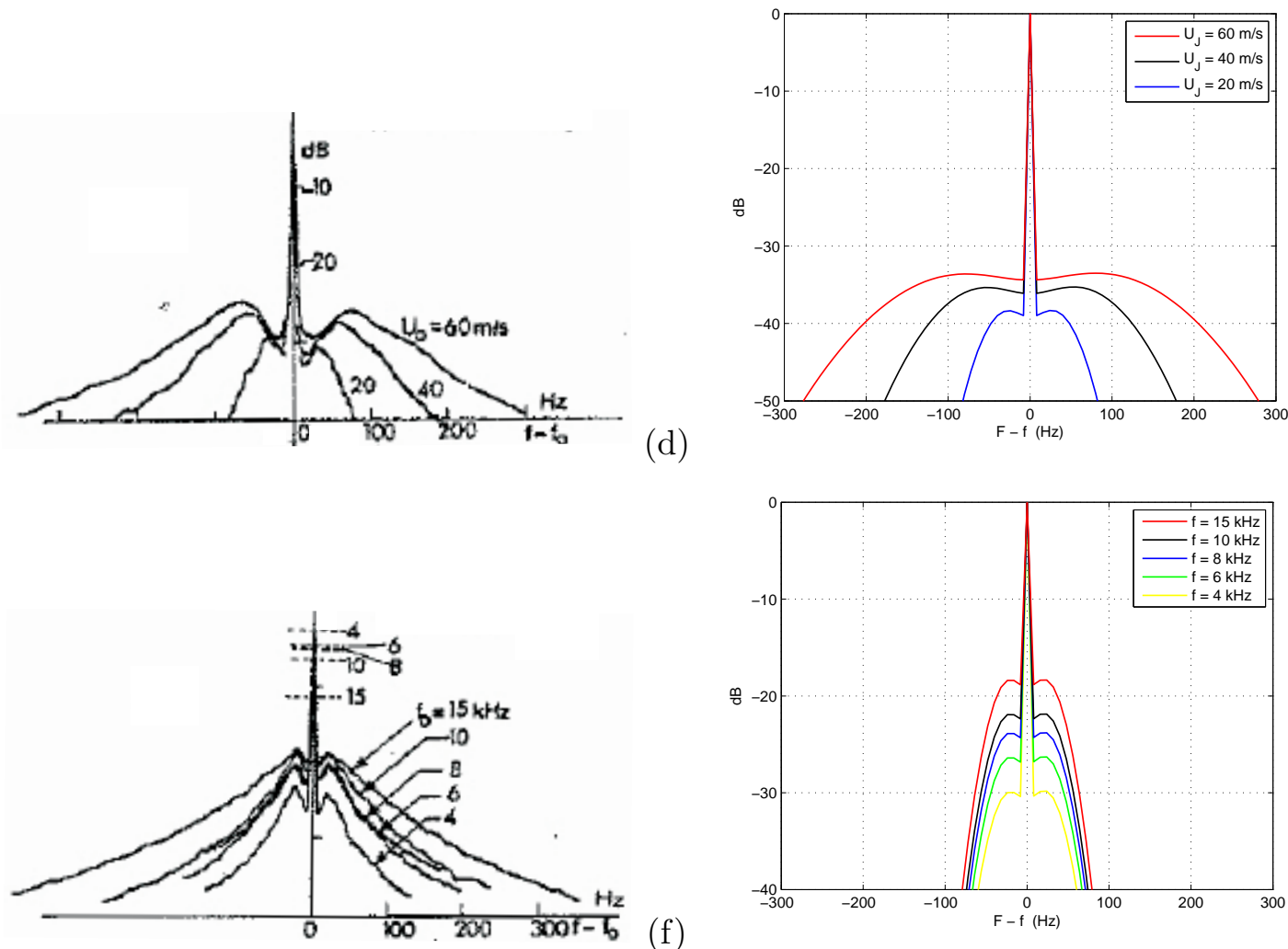

(e)

(f)

Figure 5. Scattered acoustic field at $\Theta=90^{\circ}$. Measurements and predictions of normalized farfield spectral density. Prediction uses Gaussian-HIT correlation function for frozen turbulence with $U_{c} / U_{J}=0.5$ and $l / \delta=1$. (a-b) Effect of varying source frequency at source position $x=0.5 \mathrm{~m}$. (c-d) Effect of varying jet velocity at source position $x=0.5 \mathrm{~m}$. (e-f) Effect of varying source frequency at source position $x=1.95 \mathrm{~m}$. Figures a, c, e reproduced from Candel et al. ${ }^{1}$ (p. 295, figure 25). (C)1975 IEEE

\section{2. $\Theta<90^{\circ}$}

In figure 7 a number of predicted haystacks are shown which have been calculated using the full transfer function $\mathcal{H}$ given by (25). These results pertain to one case considered in Candel et al. ${ }^{1}$ but only the scattered azimuthal order $M=0$ has been included in the simulations. Owing to this, the tone has not been included in figure 7 because the relative level of the haystack is 
(a)

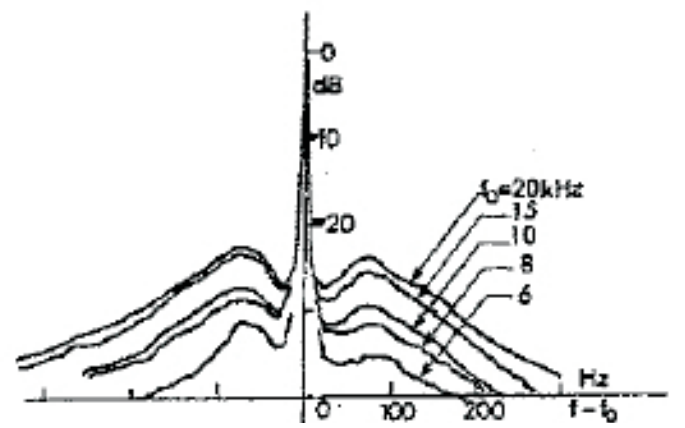

(c)

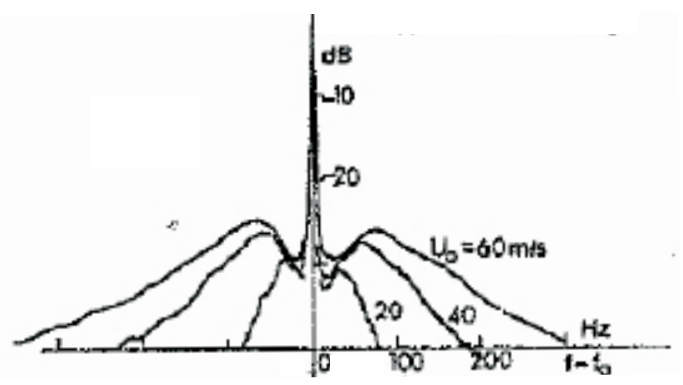

(d)

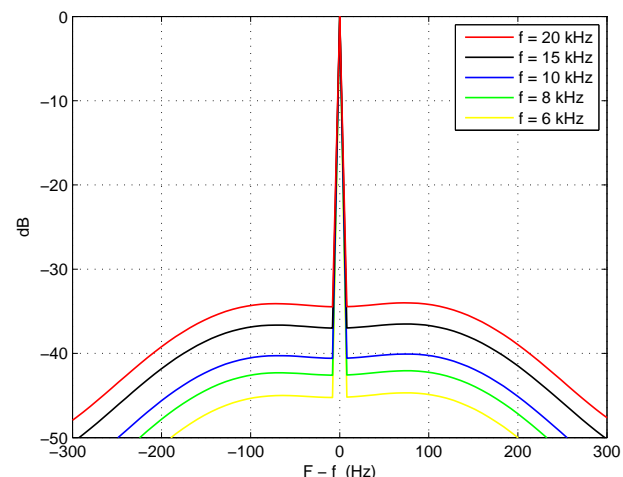

(b)
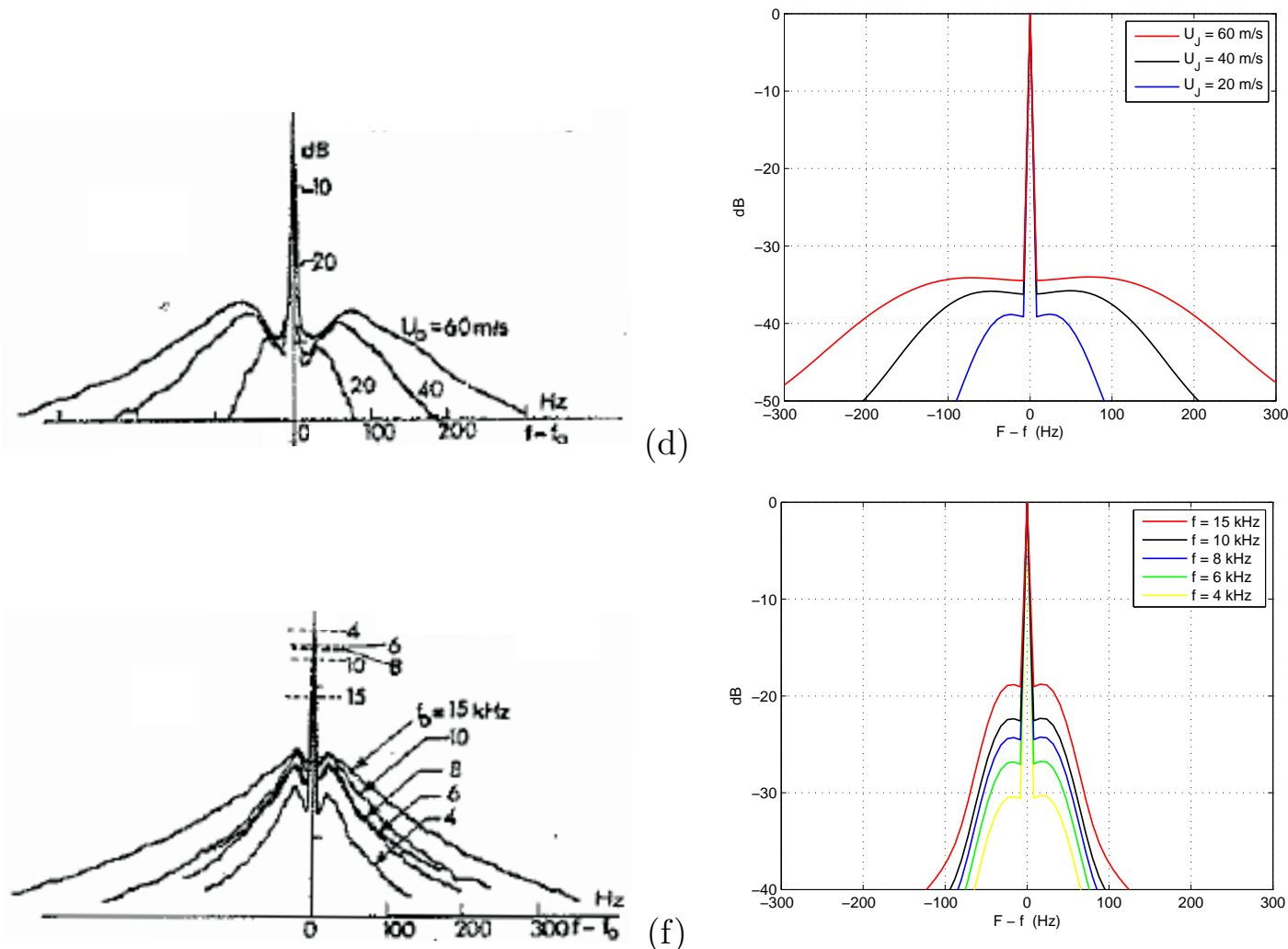

(e)

(f)

Figure 6. Scattered acoustic field at $\Theta=90^{\circ}$. Measurements and predictions of normalized farfield spectral density. Prediction uses Gaussian-HIT correlation function for non-frozen turbulence with $U_{c} / U_{J}=0.5, l / \delta=1$ and $\tau U_{c} / l=4$. (a-b) Effect of varying source frequency at source position $x=0.5 \mathrm{~m}$. (c-d) Effect of varying jet velocity at source position $x=0.5 \mathrm{~m}$. (e-f) Effect of varying source frequency at source position $x=1.95 \mathrm{~m}$. Figures a, c, e reproduced from Candel et al. ${ }^{1}$ (p. 295, figure 25). (C)1975 IEEE

about $60 \mathrm{~dB}$ lower than the tone. Predicted haystacks are shown at observer polar angles from $\Theta=90^{\circ}$ to $\Theta=20^{\circ}$. The critical angle is $31.8^{\circ}$ (see Table 1 ).

The predicted haystacks' shapes do not alter significantly in the range $50^{\circ}<\Theta \leq 90^{\circ}$, apart from the 'double-humped' shape is gradually eroded as $\Theta$ is reduced. However as $\Theta$ approaches the critical angle, the haystack level increases and the haystack shape is no longer symmetric 
about $F-f=0$. Inside the cone of silence the haystack level falls as $\Theta$ is reduced, whilst the haystack shape remains non-symmetric. At polar angles near the critical angle, the preferential scattering in the range $F-f>0$ appears to be a consequence of assuming that the incident field has uniform directivity for $\theta>\theta_{c}$ and is zero for $\theta<\theta_{c}$. Thus, the only contribution to the scattered field inside the cone of silence will be sound from the incident field outside the cone of silence that is scattered back inside the cone of silence. Predictions for the radiation of a turbine tone would require a realistic incident field directivity to be used. For a high-frequency tone radiated from a jet nozzle the assumption of weak source directivity would be unrealistic.

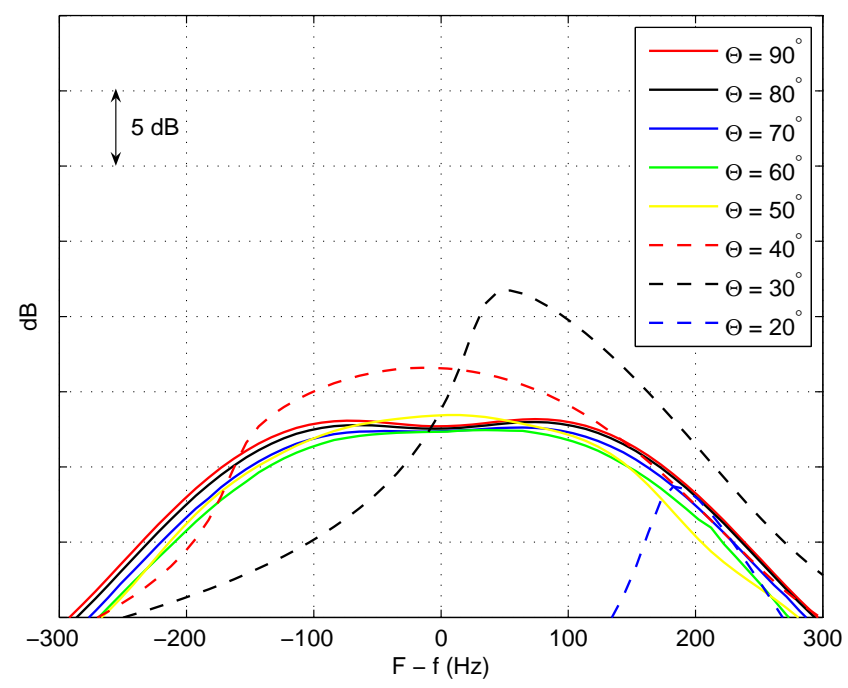

Figure 7. Effect of varying the observer polar angle from $\Theta=90^{\circ}$ to $\Theta=20^{\circ}$. Prediction uses Gaussian-HIT correlation function for non-frozen turbulence with $U_{c} / U_{J}=0.5, l / \delta=1$ and $\tau U_{c} / l=4$. Jet velocity $U_{J}=60 \mathrm{~ms}^{-1}$. Source position $x=0.5 \mathrm{~m}$. Incident acoustic field: $f=20 \mathrm{kHz}, \mathrm{m}=0$, uniform directivity. Scattered acoustic field: $M=0$ only.

\section{Parametric study}

In figures (8)-(12) the effect of varying the parameters in the model is shown for one case considered in Candel et al. ${ }^{1}$ This is the same case that was used to obtain the predictions shown for $\Theta<90^{\circ}$ in the preceding section. In figure 8 it is seen that varying the number of scattered azimuthal orders included in the prediction does not affect the haystack shape, only the absolute level.

In figure 9 there is a comparison of a haystack predicted using the full solution ( $\mathcal{H}$ given by $(25))$ and a haystack predicted using the approximate outside cone of silence solution $(\mathcal{H}$ given by (36)). There is no discernible difference in the shape of the two haystacks, but the full solution is approximately $3 \mathrm{~dB}$ higher. Presumably this is because the approximate solution neglects any reflections at the turning point. ${ }^{\mathrm{a}}$ An incoherent sum of direct and reflected rays would increase the predicted level by about $3 \mathrm{~dB}$ owing to an approximate doubling of the mean square pressure.

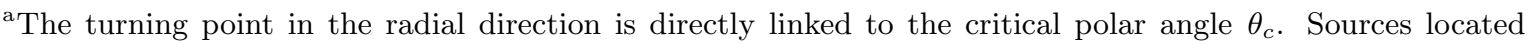
above or below the turning point are related to sound radiation above or below the critical angle (i.e. outside or inside the cone of silence).
} 
In figures (10)-(12) the effect of varying $l, \tau$ or $U_{c}$ respectively is shown. It is seen that varying these parameters in the model can affect the width of the haystack and its absolute level. In general, the haystacks become narrower as $l$ or $\tau$ are increased, or $U_{c}$ is decreased. Conversely, the haystacks become broader as $l$ or $\tau$ are decreased, or $U_{c}$ is increased. Also it appears that varying the lengthscale has the most affect on the absolute level. From (44)-(46) the turbulent velocity spectrum $\Phi_{i i} \propto l^{3}$, so increasing $l$ will increase the absolute level of the predicted haystack.

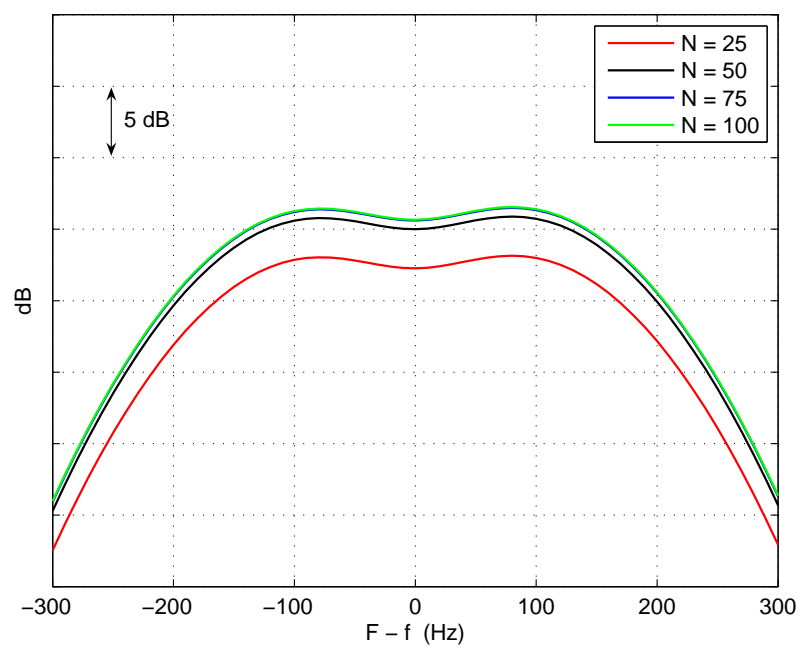

Figure 8. Effect of varying the number of scattered azimuthal orders. Scattered acoustic field at $\Theta=90^{\circ}, \sum_{M=-N}^{N}$.

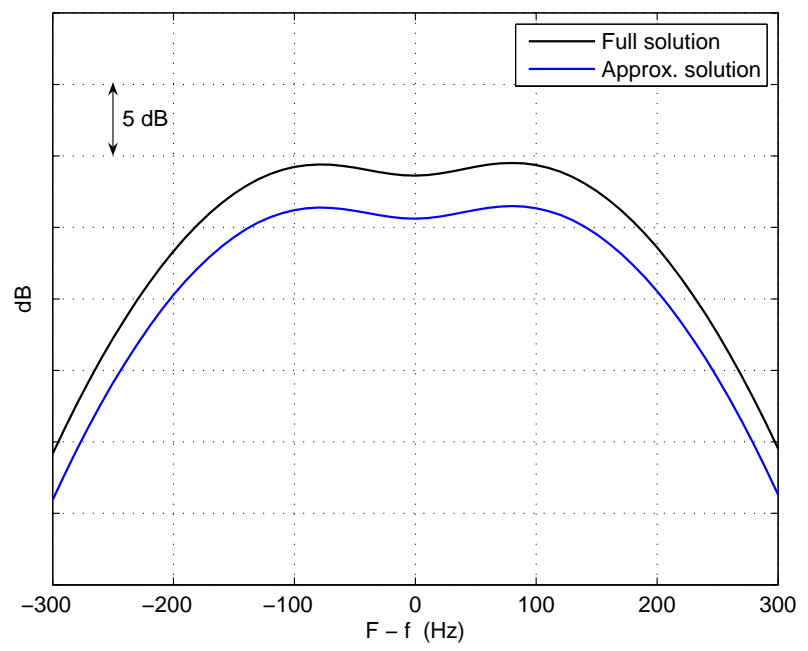

Figure 9. Comparison between the full solution and the approximate outside cone of silence solution. Scattered acoustic field at $\Theta=90^{\circ}, \sum_{M=-75}^{75}$. 


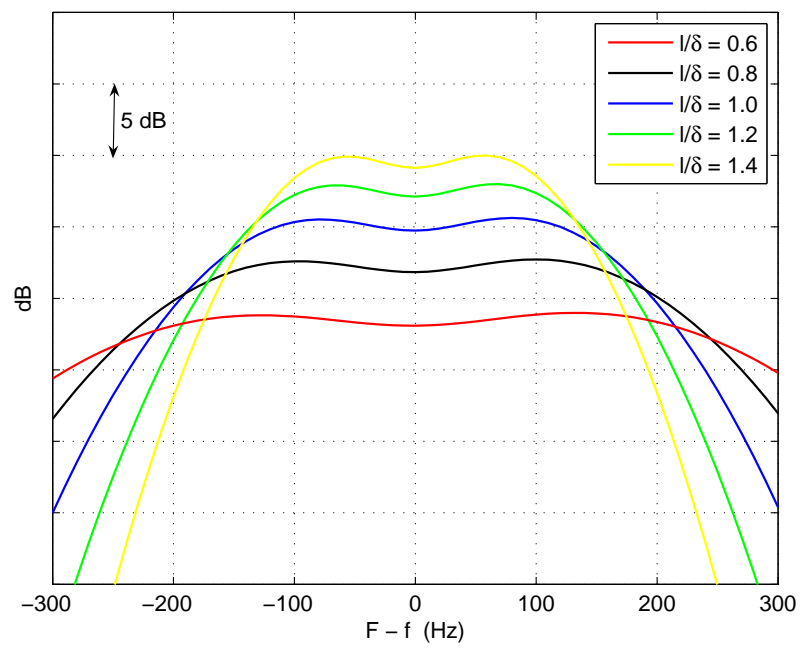

Figure 10. Effect of varying the turbulence correlation lengthscale $l$. Scattered acoustic field at $\Theta=90^{\circ}, M=0$.

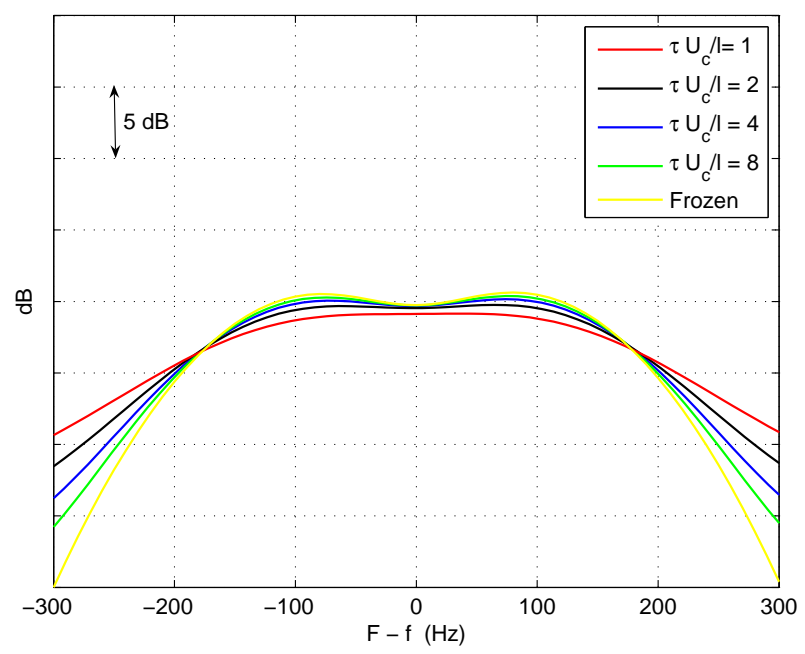

Figure 11. Effect of varying the turbulence correlation timescale $\tau$. Scattered acoustic field at $\Theta=90^{\circ}, M=0$. Note that with frozen turbulence $\tau U_{c} / l \rightarrow \infty$. 


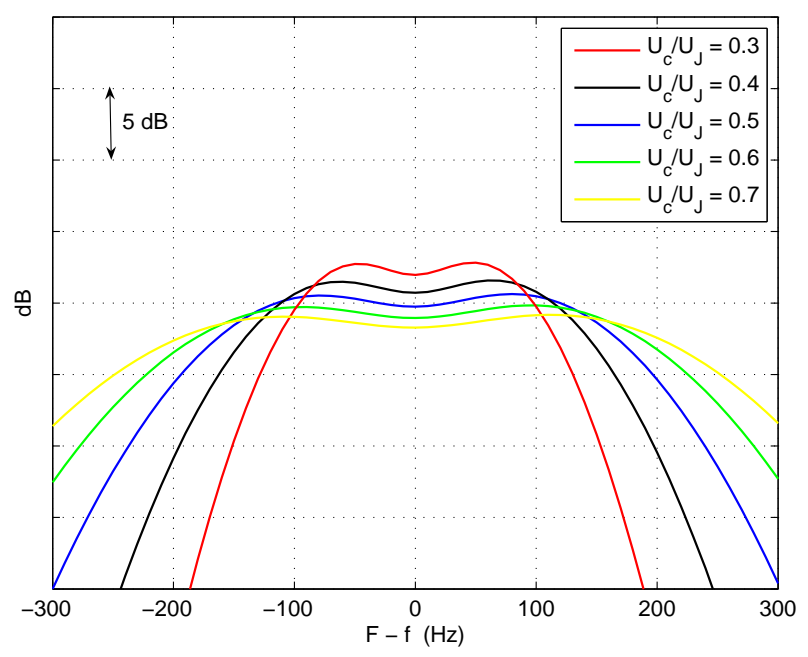

Figure 12. Effect of varying the turbulence convection velocity $U_{c}$. Scattered acoustic field at $\Theta=90^{\circ}, M=0$.

\section{Conclusions}

The spectral broadening effect of a turbulent shear layer has been modelled analytically with a high-frequency weak-scattering approach. The principal result of this work is equation (35), which gives an approximate expression of the far-field mean-square pressure directivity of the scattered acoustic field. This is expressed in terms of a transfer function $\mathcal{H}$ which links the incident and scattered fields. Two alternative transfer functions are presented. The full transfer function, given by (25), is valid to use at all polar angles, including inside the cone of silence. The simplified transfer function, given by (36), incorporates some additional approximations, resulting in a simpler function, but is valid only outside the cone of silence. Comparison between predictions, made with both frozen and non-frozen turbulence models, and experimental results, from an open flow wind tunnel, show that general trends in the data can be well predicted.

A key application of this result concerns radiation of turbine tones from the exhaust nozzle of a turbofan aero-engine: these tones propagate to the far field through one or more turbulent shear layers. In order to apply this work to turbofan aero-engines, this will require modal directivity fields to be calculated for a mode exiting a duct and propagating through a shear layer. This in itself is a non-trivial exercise. A Wiener-Hopf solution by Gabard \& Astley ${ }^{18}$ may provide suitable directivity fields, although this solution is valid for a time-harmonic mode exiting an annular duct, where there is a vortex sheet between the jet flow and the freestream. Although in this work a shear layer of finite thickness is of interest, the solution in Ref. [18] may in fact provide reasonably realistic far-field directivity functions of the incident field.

It is important to note that the result derived here is valid only in the case of 'weak' scattering. In the model this is manifested through the fact that the source terms in the scattering equation are modelled using only the incident field, so interactions between the scattered field and the turbulence are ignored. Physically, such a model will be valid in the case where, after haystacking has occured, the tone is still visible in the narrowband spectrum and is significantly higher that the broadband haystack level. However, it has been observed on certain engines at certain operating conditions that the haystacking may be so severe that the tone is no longer 
visible in the far-field spectrum, as all the tone energy has been scattered into the haystack. We refer to this as 'strong' scattering, which is beyond the limits of the current model to predict. This we intend to be an area of future work.

\section{Acknowledgments}

The authors would like to acknowledge the contributions of the late Alex M. Cargill, who originally formulated the outside cone of silence weak-scattering model used in this work while he was employed at Rolls-Royce plc.

The work was funded by the European sixth framework project TURNEX, coordinator Brian J. Tester and technical officer Daniel Chiron.

\section{References}

${ }^{1}$ S. CANDEL, A. GUEDEL, and A. JULIENNE. Refraction and scattering of sound in an open wind tunnel flow. Proceedings of the Sixth International Congress on Instrumentation in Aerospace Simulation Facilities, pages 288-300, 1975.

${ }^{2}$ A.M. CARGILL. Theory for sound scattering by turbulent shear layers. I: Derivation of an equation. Rolls-Royce Theoretical Science Group Report TSG0456, 1989.

${ }^{3}$ A.M. CARGILL. Theory for sound scattering by turbulent shear flows. II: Scattering theory. Rolls-Royce Theoretical Science Group Report TSG0457, 1989.

${ }^{4}$ D.C. MATHEWS and A.A. PERACHIO. Progress in core engine and turbine noise technology. AIAA 74-948, 1974.

${ }^{5}$ D.C. MATHEWS, R.T. NOGEL, and J.D. KESTER. Review of theory and methods for turbine noise prediction. AIAA 75-540, 1975.

${ }^{6} \mathrm{~S}$. CANDEL, A. GUEDEL, and A. JULIENNE. Radiation, refraction and scattering of acoustic waves in a free shear flow. AIAA 76-544, 1976. Proceedings of the 3rd AIAA Aeroacoustics Conference, Palo Alto, California, July 20-23, 17 pp.

${ }^{7}$ S. CANDEL, M. JULLIAND, and A. JULIENNE. Shielding and scattering by a jet flow. AIAA 76-545, 1976. Proceedings of the 3rd AIAA Aeroacoustics Conference, Palo Alto, California, July 20-23, 15 pp.

${ }^{8}$ L.M.B.C. CAMPOS. The spectral broadening of sound by turbulent shear layers. Part I. The transmission of sound through turbulent shear layers. Journal of Fluid Mechanics, 89:723-749, 1978.

${ }^{9}$ L.M.B.C. CAMPOS. The spectral broadening of sound by turbulent shear layers. Part 2. The spectral broadening of sound and aircraft noise. Journal of Fluid Mechanics, 89:751-783, 1978.

${ }^{10}$ A.M. CARGILL. Sound propagation through fluctuating flows - Its significance in aeroacoustics. AIAA 83-0697, 1983. Proceedings of the 8th AIAA Aeroacoustics Conference, Atlanta, Georgia, April 11-13, 13 pp.

${ }^{11}$ R. EWERT, O. KORNOW, B.J. TESTER, C.J. POWLES, J.W. DELFS, and M. ROSE. Spectral broadening of jet engine turbine tones. AIAA 2008-2940, 2008. Proceedings of the 14th AIAA/CEAS Aeroacoustics Conference, Vancouver, Canada, May 5-7.

${ }^{12}$ M.E. GOLDSTEIN. Aeroacoustics. McGraw-Hill, Inc., 1976.

${ }^{13}$ D.W. WUNDROW and A. KHAVARAN. On the applicability of high-frequency approximations to lilley's equation. Journal of Sound and Vibration, 272:793-830, 2004.

${ }^{14}$ G.K. BATCHELOR. The Theory of Homogeneous Turbulence. Cambridge University Press, 1953.

${ }^{15}$ R. EWERT. Broadband slat noise predictions based on CAA and stochastic sound sources from fast random-particle mesh (RPM) method. Computers and Fluids, 37:369-387, 2008.

${ }^{16}$ P.J. MORRIS and S. BOLURIAAN. The prediction of jet noise from CFD data. AIAA 2004-297r, 2004. Proceedings of the 10th AIAA/CEAS Aeroacoustics Conference, Manchester, UK, May 10-12, $11 \mathrm{pp}$.

${ }^{17}$ M. HARPER-BOURNE. Jet noise turbulence measurements. AIAA 2003-3214, 2003. Proceedings of the 9th AIAA/CEAS Aeroacoustics Conference \& Exhibit, Hilton Head, South Carolina, May 12-14, 14 pp.

${ }^{18}$ G. GABARD and R.J. ASTLEY. Theoretical model for sound radiation from annular jet pipes: far- and near-field solutions. 549:315-341, 2005. Journal of Fluid Mechanics.

${ }^{19}$ B.J. TESTER and C.L. MORFEY. Developments in jet noise modelling - Theoretical predictions and comparisons with meassured data. Journal of Sound and Vibration, 46(1):79-103, 1976. 


\section{Appendix: Source terms in the approximate form of Lilley's equation}

After considerable algebraic manipulations it can be shown that the source terms in (8) not involving gradients of mean flow quantities are

$$
\begin{aligned}
& \bar{\rho} \frac{\overline{\mathrm{D}}}{\mathrm{D} t}\left[\frac{\partial^{2}}{\partial x^{2}}\left(u_{x}^{\prime} u_{x}^{\prime}\right)+\frac{\partial^{2}}{\partial r^{2}}\left(u_{r}^{\prime} u_{r}^{\prime}\right)+\frac{2}{r} \frac{\partial}{\partial r}\left(u_{r}^{\prime} u_{r}^{\prime}\right)+\frac{1}{r^{2}} \frac{\partial^{2}}{\partial \phi^{2}}\left(u_{\phi}^{\prime} u_{\phi}^{\prime}\right)-\frac{1}{r} \frac{\partial}{\partial r}\left(u_{\phi}^{\prime} u_{\phi}^{\prime}\right)\right. \\
+ & \left.2 \frac{\partial^{2}}{\partial x \partial r}\left(u_{x}^{\prime} u_{r}^{\prime}\right)+\frac{2}{r} \frac{\partial}{\partial x}\left(u_{x}^{\prime} u_{r}^{\prime}\right)+\frac{2}{r} \frac{\partial^{2}}{\partial x \partial \phi}\left(u_{x}^{\prime} u_{\phi}^{\prime}\right)+\frac{2}{r} \frac{\partial^{2}}{\partial r \partial \phi}\left(u_{r}^{\prime} u_{\phi}^{\prime}\right)+\frac{2}{r^{2}} \frac{\partial}{\partial \phi}\left(u_{r}^{\prime} u_{\phi}^{\prime}\right)\right] \\
- & \bar{\rho} \overline{\mathrm{D}}_{p}^{2} \frac{\partial}{\mathrm{D} t^{2}}\left[\frac{\partial}{\partial x}\left(s^{\prime} u_{x}^{\prime}\right)+\frac{\partial}{\partial r}\left(s^{\prime} u_{r}^{\prime}\right)+\frac{1}{r}\left(s^{\prime} u_{r}^{\prime}\right)+\frac{1}{r} \frac{\partial}{\partial \phi}\left(s^{\prime} u_{\phi}^{\prime}\right)\right]
\end{aligned}
$$

when expressed in cylindrical polar coordinates. It is noted that the double divergence term $\partial^{2}\left(u_{i}^{\prime} u_{k}^{\prime}\right) / \partial x_{i} \partial x_{k}$ in (8), expressed in cylindrical polar coordinates, was derived originally by Tester \& Morfey. ${ }^{19}$ 